\title{
Preparation and Characterization of 5-Fluorouracil Loaded Chitosan Microspheres by a Two-Step Solidification Method
}

\author{
Yi Sun, Lei Gu, Yuan GaO, and Feng GAO* \\ Department of Pharmaceutics, School of Pharmacy, East China University of Science and Technology; 130 Meilong Road, \\ Shanghai 200237, China. Received December 25, 2009; accepted March 31, 2010; published online April 20, 2010
}

\begin{abstract}
Chitosan, as a natural biopolymer, has potential applications in controlling the release of drugs due to its unique natural and physiochemical properties. Conventionally, an emulsion crosslinking method was used to prepare chitosan microspheres. In this study, a novel method of preparing 5-fluorouracil-loaded chitosan microspheres was established by combining the emulsification technique and a two-step solidification method using both glutaraldehyde (GTA) and tripolyphosphate (TPP) as crosslinking agents. The microspheres were characterized by surface morphology, encapsulation efficiency, yield of microspheres and drug release profile in vitro. The optimal chitosan microspheres were achieved by crosslinking chitosan with both TPP $(0.1 \%, \mathrm{pH} 4.0)$ and GTA $(1.0 \%, v / v)$ with span-80 additive under a crosslinking time of $1 \mathrm{~h}$ with TPP and GTA, respectively.
\end{abstract}

Key words chitosan microsphere; 5-fluorouracil; two-step solidification; glutaraldehyde; tripolyphosphate

Microparticles and nanoparticles have been widely studied for applications in drug release systems. To prevent a drug from rapid release, the use of biodegradable polymers which serve as protective drug coatings has been developed. ${ }^{1)}$ Chitosan, as a biodegradable and biocompatible polymer, is extensively used as a carrier for encapsulation of drugs and biological substances in the pharmaceutical industry ${ }^{2)}$ due to: its ability in a drug controlled-release system, its solubility in aqueous acidic solution which avoids the use of hazardous organic solvents while fabricating particles, its cationic nature that allows ionic to crosslink with multivalent anions, the capacity of chemical crosslink applied by its amino groups and its mucoadhesive character of increasing residual time at the site of absorption. ${ }^{3)}$ Emulsion crosslinking, coacervation/precipitation, spray-drying and ionic gelation methods are usually used in preparing chitosan microspheres. Water-soluble drugs can be loaded by using the emulsion crosslinking technique with high encapsulation efficiency. In this method, a water/oil (W/O) emulsion was prepared by emulsifying the chitosan aqueous solution in the oil phase and aqueous droplets are stabilized by using a suitable surfactant. Then the stable emulsion is solidified by an appropriate crosslinking agent such as glutaraldehyde (GTA) or tripolyphosphate (TPP) to harden the droplets. ${ }^{4)}$

5-Fluorouracil (5-FU), a water soluble drug, is one of the most effective chemotherapeutic agents with clinical activity against squamous esophageal carcinoma. It can cause severe gastrointestinal, hematological, neural, cardiac and dermatological toxic effects when administered intravenously. ${ }^{5)}$ The systemic toxic effects and very short plasma half-life (approximately $11 \mathrm{~min}$ ) make it particularly suitable to be delivered by a local drug delivery system which can provide a continuous sustained release. ${ }^{6}$ Encapsulation in microspheres might be able to extend its biological availability.

This research aims at the production of chitosan microspheres for the controlled delivery of 5-FU. According to previous studies, drug release from chitosan microspheres could be well controlled by crosslinking the matrix using chemical crosslinking agents such as GTA. ${ }^{7-10)}$ However, a high burst effect was found in GTA crosslinked microspheres ${ }^{11,12)}$ and chemically synthesized GTA can cause irritation to mucosal membranes due to its toxicity. In order to overcome the disadvantages of chemical crosslinking, an ionic crosslinking method has been applied. ${ }^{13)}$ Among the available multivalent anions, the sodium TPP is nontoxic and efficient in forming chitosan microspheres by ionic interactions. ${ }^{14)}$ However, ionic crosslinking interaction also has drawbacks. Since the interactions between chitosan and anion crosslinking agents highly depend on the molecular structure of the anions, ${ }^{15}$ ) the charge density of anion crosslinking agents ${ }^{16}$ as well as the $\mathrm{pH}$ values of solutions, ${ }^{17)}$ ionotropic crosslinked chitosan microspheres have poor mechanical strength which results in morphological distortion in the subsequent washing and drying process. ${ }^{3)}$

Here we describe a feasible two-step solidification method for preparation of 5-FU-loaded chitosan microspheres which was developed to improve the poor mechanical strength of ionotropic crosslinked chitosan microspheres. This was achieved by optimizing the solidification procedure by using both GTA and TPP as crosslinking agents. The effects of solidification conditions on surface morphology, encapsulation efficiency, yield of microspheres and drug release profile in vitro were investigated.

\section{Experimental}

Materials Chitosan (Mw: 1000000) with $89 \%$ deacetylation degree was purchased from Yuanju Bio-tech Co., Ltd. (Shanghai, China). 5-Fluorouracil (5-FU) was provided by Linyi Furui Fine Chemical Industry Co., Ltd. (Shandong, China). Tripolyphosphate (TPP) was purchased from Wuxi Jierui Chemistry Industry Co., Ltd. (Wuxi, China). Glutaradehyde (GTA) was obtained as a $25 \%$ aqueous solution from Chinese Medicine Group Shanghai Chemical Reagent Company (Shanghai, China). All other reagents were of analytical grade.

Preparation of Chitosan Microspheres 5-FU-loaded chitosan microspheres were prepared by emulsion crosslinking technique. Firstly, chitosan was dissolved in $2 \%(\mathrm{v} / \mathrm{v})$ acetic acid solution to obtain a polymer solution at a concentration of $2.5 \%(\mathrm{w} / \mathrm{v})$. 5 -FU was dissolved directly into the chitosan solution to form a drug/polymer solution (aqueous phase) at a final concentration of $2.5 \%(\mathrm{w} / \mathrm{v})$. This aqueous solution $(2 \mathrm{ml})$ was emulsified into $20 \mathrm{ml}$ liquid paraffin (oil phase) containing $1 \%(\mathrm{v} / \mathrm{v})$ different additives (span-80, tween-80, F68, PEG-6000) by using a homogenizer (BME100LXS, Weiyu Co., Ltd., Shanghai, China) and stirred at $2000 \mathrm{rpm}$ for $0.5 \mathrm{~h}$ to form a W/O emulsion. At the end of emulsification, the emulsion was solidified by crosslinking process using TPP or/and GTA as the crosslinking agent. Briefly, the emulsion was first gelated by dropping TPP aqueous solution $(0.00-0.15 \%, \mathrm{pH} 2-6)$ at $300 \mathrm{rpm}$ for $1 \mathrm{~h}$ (the dropping rate was 
$0.2 \mathrm{ml} / \mathrm{min})$, then a definite amount of GTA $(0.0-3.0 \%, \mathrm{v} / \mathrm{v})$ was dropped to crosslink the gel at a speed of $500 \mathrm{rpm}$ for a period of time $(0.0-1.5 \mathrm{~h})$. Finally, the 5-FU-loaded chitosan microspheres were collected by pressure reduction filtration, separately washed with petroleum ether and distilled water, and then were lyophilized.

Encapsulation Efficiency and Yield of Chitosan Microspheres The encapsulation efficiency was determined as follows. Microspheres (3 mg) were dissolved completely in $50 \mathrm{ml} 0.1 \mathrm{~N} \mathrm{HCl}$ at room temperature after $1 \mathrm{~h}$ in an ultrasonic washing unit (KLF-X, Zhangjiagang Keli Ultrasonic Co., Ltd., Zhang Jia Gang, China), then were centrifuged at $3000 \times \boldsymbol{g}$ for $30 \mathrm{~min}$ and filtered ( $0.2 \mu \mathrm{m}$ nylon filters, Whatman Co., Ltd., U.K.). The fluid was assayed for drug content by UV spectroscopy (751-GW, Shanghai Precision and Scientific Instrument Co., Ltd., Shanghai, China) at the wavelength of $266 \mathrm{~nm}$ and calculated by interpolation from an according standard calibration curve. Encapsulation efficiency was calculated from the actual drug loading of the microspheres and the theoretical drug loading.

Yield of the preparation was determined by the weight of the products, freeze-dried microspheres, with respect to the weight of the initial polymer and the drug used. All samples were analyzed in triplicate.

$$
\begin{aligned}
\text { yield }(\%)= & (\text { mass of microspheres } /(\text { mass of drug }+ \text { mass of polymer })) \\
& \times 100 \%
\end{aligned}
$$

encapsulation efficiency $(\%)=($ actual drug loading/theoretical drug loading) $\times 100 \%$

In Vitro Drug Release of Chitosan Microspheres Microspheres $(10 \mathrm{mg}$ ) were suspended in $100 \mathrm{ml}$ of release media (phosphate buffered saline (PBS), pH 7.4). At predetermined intervals of $48 \mathrm{~h}, 4 \mathrm{ml}$ of the release buffer was sampled and replaced with fresh PBS buffer. The samples were diluted and analyzed by UV spectroscopy. The percentage of cumulative release of 5-FU was obtained from the standard calibration curve. All experiments were performed in triplicate.

The drug released from the microspheres is expressed as the cumulative release by the following equation:

$$
\text { cumulative release }(\%)=\frac{V_{\mathrm{e}} \sum_{i=1}^{n-1} C_{i}+V_{\mathrm{o}} C_{n}}{W_{\mathrm{o}}} \times 100 \%
$$

where $V_{\mathrm{e}}$ is the replaced volume of PBS buffer $(4 \mathrm{ml}), V_{\mathrm{o}}$ is the total volume of PBS buffer $(100 \mathrm{ml}), C_{i}$ and $C_{n}$ are the drug release concentration $(\mu \mathrm{g} / \mathrm{ml})$ and $W_{\mathrm{o}}$ is the amount of the drug loaded onto the microspheres $(\mu \mathrm{g})$.

Scanning Electron Microscope Analysis Surface morphology of 5FU-loaded chitosan microspheres after drying was observed by scanning electron microscopy (SEM). Microspheres were freeze-dried, coated with platinum and observed with a scanning electron microscope (JSM-6360LV,
JEOL Ltd., Tokyo, Japan).

Particle Size Distribution Analysis The particle size distribution of 5FU-loaded chitosan microspheres after drying was measured by laser diffractometry. Freeze-dried microspheres were redispersed in distilled water and sized by a laser diffraction particle size analyzer (Mastersizer 2000, Malvern Instruments Ltd., U.K.).

Fourier Transform Infrared Spectral Studies of Chitosan Microspheres Fourier Transform Infrared (FT-IR) spectra of 5-FU-loaded chitosan microspheres were recorded on $\mathrm{KBr}$ pellets with a FT-IR spectrophotometer (FT-IR1730, Perkin-Elmer Inc., U.S.A.).

\section{Results and Discussion}

Effects of pH Values of TPP Solution The 5-FU-loaded chitosan microspheres were first solidified using TPP solution with different $\mathrm{pH}$ values of 2,4 and 6 , respectively, and the effects of $\mathrm{pH}$ values of the solution on encapsulation efficiency, microsphere morphology and release profile in vitro were investigated (Table 1, Fig. 1). For the two-step solidification, microspheres were formed through reactions between amino groups of chitosan and TPP ions $\left(\mathrm{HP}_{3} \mathrm{O}_{10}^{4-}\right.$ and

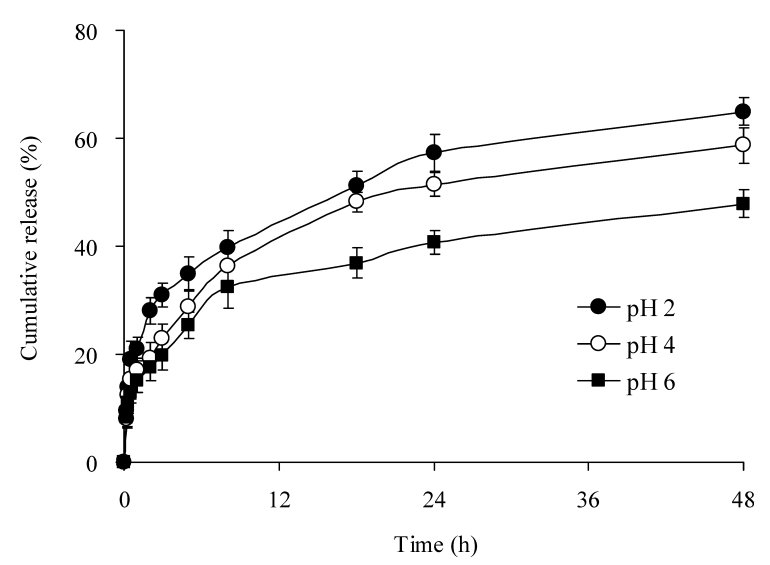

Fig. 1. Effect of $\mathrm{pH}$ Values of TPP Solution on Release Profiles in Vitro

$\mathrm{pH}$ value of TPP solution was from 2 to 6 . Other preparative conditions were controlled as follows: $0.1 \%$ TPP, $1 \mathrm{~h}$ of crosslinking time of $1.0 \%$ GTA, $1 \%$ span- 80 as ad-

\begin{tabular}{|c|c|c|c|c|c|c|}
\hline $\begin{array}{l}\mathrm{pH} \text { value of } \\
\text { TPP solution }\end{array}$ & ТPР (\%) & GTA $(\%)$ & $\begin{array}{c}\text { Crosslinking } \\
\text { time of GTA }(h)\end{array}$ & Type of additives & $\begin{array}{l}\text { Encapsulation } \\
\text { efficiency }(\%)\end{array}$ & Morphology \\
\hline 2 & 0.1 & 1.0 & 1 & span-80 & $36.3 \pm 3.5$ & Unformed \\
\hline 4 & 0.1 & 1.0 & 1 & span-80 & $78.5 \pm 1.8$ & Spherical \\
\hline 6 & 0.1 & 1.0 & 1 & span-80 & $45.1 \pm 4.4$ & Spherical \\
\hline $4^{a)}$ & 0.0 & 1.0 & 1 & span-80 & $50.4 \pm 2.6$ & Spherical \\
\hline 4 & 0.05 & 1.0 & 1 & span-80 & $70.6 \pm 2.3$ & Spherical \\
\hline 4 & 0.1 & 1.0 & 1 & span-80 & $78.5 \pm 1.8$ & Spherical \\
\hline 4 & 0.15 & 1.0 & 1 & span-80 & $72.4 \pm 3.4$ & Spherical \\
\hline $4^{b)}$ & 0.1 & 0.0 & - & span-80 & $77.8 \pm 3.8$ & Spherical \\
\hline 4 & 0.1 & 0.5 & 1 & span-80 & $35.4 \pm 5.6$ & Unformed \\
\hline 4 & 0.1 & 1.0 & 1 & span-80 & $78.5 \pm 1.8$ & Spherical \\
\hline 4 & 0.1 & 3.0 & 1 & span-80 & $76.7 \pm 2.3$ & Spherical \\
\hline 4 & 0.1 & 1.0 & 0.5 & span-80 & $32.3 \pm 1.9$ & Unformed \\
\hline 4 & 0.1 & 1.0 & 1 & span-80 & $78.5 \pm 1.8$ & Spherical \\
\hline 4 & 0.1 & 1.0 & 1.5 & span-80 & $74.7 \pm 3.7$ & Spherical \\
\hline 4 & 0.1 & 1.0 & 1 & none & $29.2 \pm 4.5$ & Unformed \\
\hline 4 & 0.1 & 1.0 & 1 & tween- 80 & $53.1 \pm 4.1$ & Spherical \\
\hline 4 & 0.1 & 1.0 & 1 & F68 & $38.7 \pm 3.8$ & Unformed \\
\hline 4 & 0.1 & 1.0 & 1 & PEG-6000 & $45.1 \pm 2.9$ & Unformed \\
\hline 4 & 0.1 & 1.0 & 1 & span-80 & $78.5 \pm 1.8$ & Spherical \\
\hline
\end{tabular}
ditive $(n=3)$.

Table 1. Effects of Preparation of 5-FU-Loaded Chitosan Microspheres on Encapsulation Efficiency and Morphology $(n=3)$

a) The conventional crosslinking method using GTA only. b) The conventional crosslinking method using TPP only. Other formulations were all prepared by a two-step solidification method. 
$\mathrm{P}_{3} \mathrm{O}_{10}^{5-}$ ), aldehyde groups of GTA. In the first solidification step, TPP reacted with amino groups of chitosan to form a gel. The ionization degree of TPP was dependent on the $\mathrm{pH}$ value of solution. In acidic solution, though the gel was completely ionic crosslinked, ${ }^{12)}$ the mechanical strength of the ionic crosslinking gel was still quite poor. Therefore, the gel was further solidified by the reaction between aldehyde groups of GTA and amino groups of chitosan. The reactivity between aldehyde groups and amino groups is also highly dependent on $\mathrm{pH}$ value, and shows a low reactivity under acidic conditions. Thus, when the $\mathrm{pH}$ value of TPP solution was 2, the first solidification step of gel formation predominated. In the subsequent washing process, the drug was easily washed out, resulting in a low encapsulation efficiency of $36.3 \%$. Contrarily, when $\mathrm{pH}$ value of TPP solution was higher $(\mathrm{pH} \mathrm{6)}$, the second solidification step (GTA reacted with chitosan) became predominant. Microspheres with a spherical and smooth surface were formed. Significant contraction of gels was observed during the reaction between GTA and chitosan, which induced the leakage of drug from the microspheres; the contraction led to a low encapsulation efficiency. When the $\mathrm{pH}$ value of TPP solution was 4, the encapsulation efficiency of 5-FU-loaded microspheres went up greatly to $78.5 \%$. The first solidification step worked well for gel formation that effectively prevented drug leakage, and the proper chemical crosslinking in the second solidification step contributed to the formation of spherical and smooth microspheres that prevented the drug from being washed out of microspheres.

Figure 1 shows the release profiles of 5-FU from chitosan microspheres at different $\mathrm{pH}$ values of TPP solution. With the increase of $\mathrm{pH}$ value, the structure of microspheres became denser, which adversely slowed drug release. Therefore, the $\mathrm{pH}$ value of TPP solution played an important role in preparation of 5-FU-loaded chitosan microspheres by a two-step solidification method. Considering the effects of $\mathrm{pH}$ values of TPP solution on encapsulation efficiency, microsphere morphology and release profile in vitro, the optimal $\mathrm{pH}$ value of this solution was 4 .

Effects of TPP Concentration at the First Solidification Step The effects of TPP concentration on encapsulation efficiency, microsphere morphology and release profile in vitro

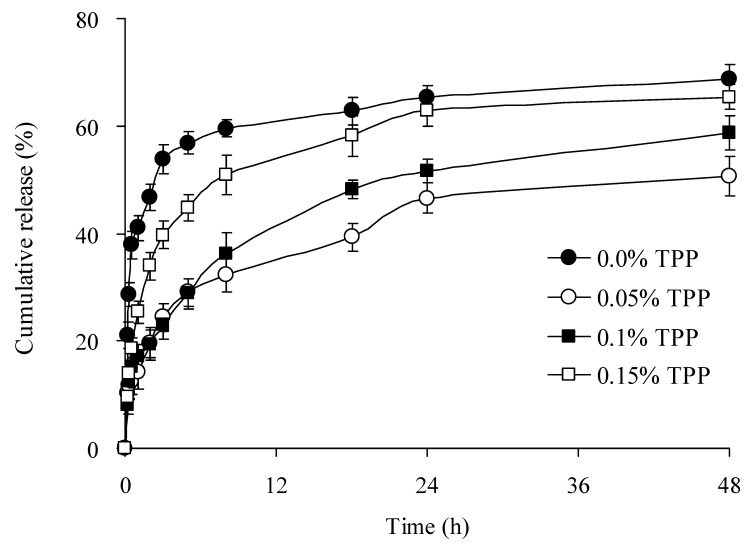

Fig. 2. Effect of TPP Concentration at the First Solidification Step on Release Profiles in Vitro

TPP concentration was from 0.00 to $0.15 \%$. Other preparative conditions were controlled as follows: $\mathrm{pH} 4$ of TPP solution, $1 \mathrm{~h}$ of crosslinking time of $1.0 \%$ GTA, $1 \%$ span- 80 as additive $(n=3)$. are shown in Table 1 and Fig. 2. In the absence of TPP, no well-formed gel was observed. Drug leakage was found frequently during the GTA solidification process, leading to a low encapsulation efficiency $(50.4 \%)$. In the two-step solidification process, the concentration of TPP was changed from 0.05 to $0.15 \%$ to gelate chitosan droplets and the droplets were subsequently solidified by GTA. The encapsulation efficiencies of 5-FU-loaded chitosan microspheres using the two-step solidification method were all above $70 \%$ (Table 1).

Figure 2 shows that the microspheres crosslinked by GTA only (TPP concentration was $0.0 \%$ ) were apparently degraded and had a high burst effect. The cumulative release was much slower for microspheres which were solidified by both TPP and GTA in comparison to those that were solidified only by GTA. The increase of TPP concentration (from 0.05 to $0.15 \%$ ) accelerated the drug release from chitosan microspheres, because the activity of reaction between TPP and chitosan was proportional to the amount of TPP. At the same time, due to a large consumption of $-\mathrm{NH}_{2}$ on chitosan by interaction with TPP, the degree of crosslinking between chitosan and GTA was decreased, which resulted in a faster release of 5-FU from the microspheres. Taking all these factors into account (the microsphere morphology, encapsulation efficiency and release profile in vitro), a TPP concentration of $0.1 \%$ was selected for the solidification step.

Effects of GTA Concentration at the Second Solidification Step Different concentrations of GTA $(0.0,0.5,1.0$, $3.0 \%$ ) were used to solidify chitosan gel in the second solidification step. Effects of its concentration on encapsulation efficiency, microsphere morphology and release profile in vitro are listed in Table 1 and Fig. 3. In the two-step solidification, microspheres were easily deformed when the concentration of GTA was less than $0.5 \%$. This was caused by the GTA amount being inadequate and failing to solidify the gel. The first solidification step then became predominant, which caused the formed gel to be easily broken in the subsequent treatment. Therefore, the encapsulation efficiency was only $35.4 \%$ (Table 1).

The release profiles of 5-FU-loaded chitosan microspheres are shown in Fig. 3. Obviously, both faster drug release rate and higher burst rate were found for microspheres prepared without GTA. As shown, the release profile was greatly im-

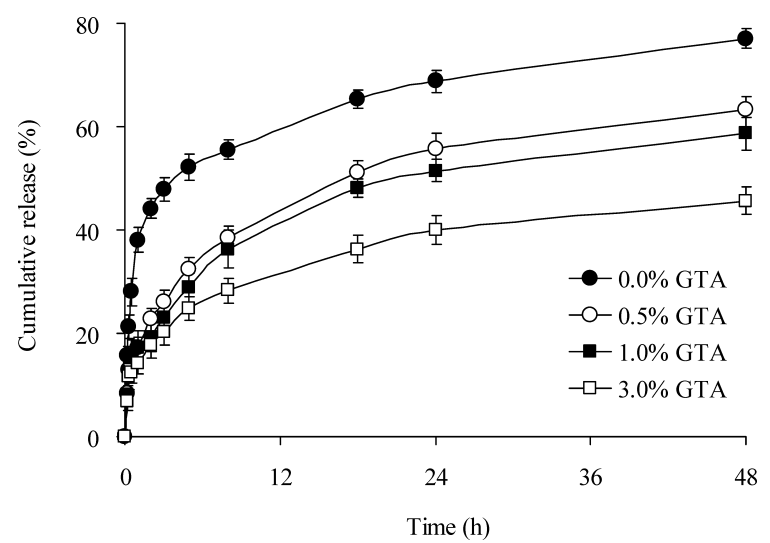

Fig. 3. Effect of GTA Concentration at the Second Solidification Step on Release Profiles in Vitro

GTA concentration was from 0.0 to $3.0 \%$. Other preparative conditions were controlled as follows: $\mathrm{pH} 4$ of $0.1 \%$ TPP solution, $1 \mathrm{~h}$ of crosslinking time of GTA, $1 \%$ span- 80 as additive $(n=3)$. 
proved with no apparent burst effect when GTA chemical solidification was applied after TPP ionic gelation. The drug release rate went down when the concentration of GTA went up.

In terms of the comprehensive effects of GTA concentration on microsphere morphology, encapsulation efficiency and release profile in vitro, the optimal concentration of GTA was $1.0 \%$.

Effects of Crosslinking Time of GTA at the Second Solidification Step In two-step solidification, chitosan microspheres were further solidified by GTA for different periods $(0.5,1.0,1.5 \mathrm{~h})$. Encapsulation efficiency, microsphere morphology and release profile in vitro were investigated to elucidate the effects of crosslinking time of GTA (Table 1, Fig. 4). When this crosslinking time was $0.5 \mathrm{~h}$, the reaction degree of amino group and aldehyde group was not efficient in solidifying the formed gel, but may have competitively destroyed the network structure of TPP-crossed microspheres, leading to fragmentation of the gel during the second solidification process. This could be the main reason for the lower encapsulation efficiency shown in Table 1 and may have accounted for the faster release in vitro shown in Fig. 4. A significant slowdown of the drug release rate was observed by further increasing crosslinking time to $1 \mathrm{~h}$. As a result, the optimal crosslinking time of GTA was around $1 \mathrm{~h}$.

Effects of Additives of Aqueous Phase Four different additives (tween-80, F68, PEG-6000 and span-80) were added into the aqueous phase, respectively, in order to improve the formation of microspheres. The effects of additives on encapsulation efficiency, microsphere morphology, yield and release profile in vitro are shown in Table 1, Table 2 and

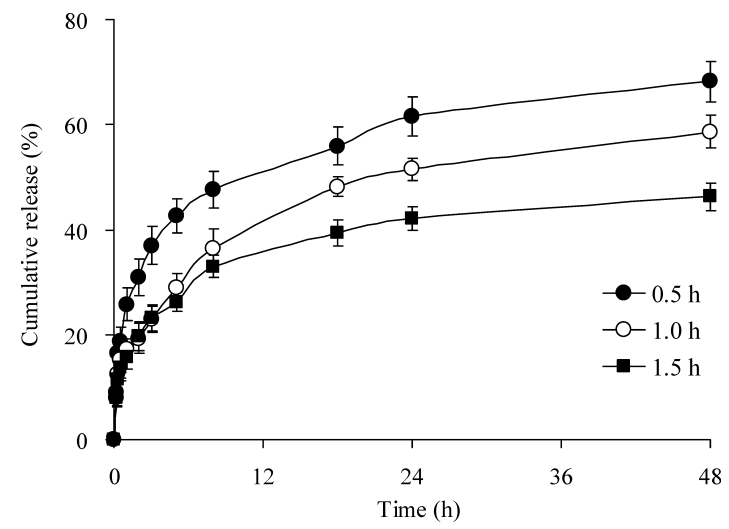

Fig. 4. Effect of Crosslinking Time of GTA at the Second Solidification Step on Release Profiles in Vitro

Crosslinking time of GTA was from $0.0-1.5 \mathrm{~h}$. Other preparative conditions were controlled as follows: $\mathrm{pH} 4$ of $0.1 \%$ TPP solution, $1.0 \%$ GTA, $1 \%$ span- 80 as additive $(n=3)$

Table 2. Yield of 5-FU-Loaded Chitosan Microspheres Using Different Types of Additives $(n=3)$

\begin{tabular}{cc}
\hline \hline Type of additives & Yield of microspheres (\%) \\
\hline None & $23.1 \pm 1.2$ \\
tween-80 & $54.3 \pm 2.3$ \\
F68 & $32.9 \pm 1.6$ \\
PEG-6000 & $35.7 \pm 2.6$ \\
span-80 & $90.2 \pm 4.7$ \\
\hline
\end{tabular}

The concentrations of all additives were $1 \%$. Other preparative conditions were controlled as follows: $\mathrm{pH} 4$ of $0.1 \%$ TPP and $1 \mathrm{~h}$ of crosslinking time of $1.0 \%$ GTA.
Fig. 5. The results suggest that only span- 80 is favorable for preparation of 5-FU-loaded chitosan microspheres. One possible reason for its good effects may come from its suitable hydrophile-lipophile balance (HLB) value. Commonly, the best value range of HLB to prepare a stable $\mathrm{W} / \mathrm{O}$ emulsion is between 3 and 6 . The value of span-80 (HLB of 4.3) is within the optimal range, while that of other additives (HLB of $15,19,29$ for tween-80, PEG-6000, F68, respectively) is far beyond the advisable range. Once a stable W/O emulsion is created, microspheres after the crosslinking process may be well formed with high drug loading and slow drug release rate.

Physicochemical and Morphological Characterization of 5-FU-Loaded Chitosan Microspheres after Optimized Preparation FT-IR spectra of 5-FU solution, together with 5-FU-loaded chitosan microspheres prepared by three methods (two-step solidification, TPP crosslinking and GTA crosslinking) are shown in Fig. 6. Figure 6a shows that the characteristic absorption peaks of 5-FU were found at $1655.80 \mathrm{~cm}^{-1}$ (overlap absorption peak of $\mathrm{C}=\mathrm{O}, \mathrm{C}=\mathrm{C}$ stretching vibration), $1432.30 \mathrm{~cm}^{-1}(\mathrm{C}-\mathrm{H}$ in-plane bending vibration of $\mathrm{CF}=\mathrm{CH}$ group), $1248.46 \mathrm{~cm}^{-1}(\mathrm{C}-\mathrm{N}$ stretching vibration), $816.78 \mathrm{~cm}^{-1}$ and $760.72 \mathrm{~cm}^{-1}$ (C-H out-of-plane formation vibration of $\mathrm{CF}=\mathrm{CH}$ group). For GTA crosslinked chitosan microspheres (Fig. 6b), the characteristic absorption peaks appeared at $1672.69 \mathrm{~cm}^{-1}(\mathrm{C}=\mathrm{N}$ stretching vibration,

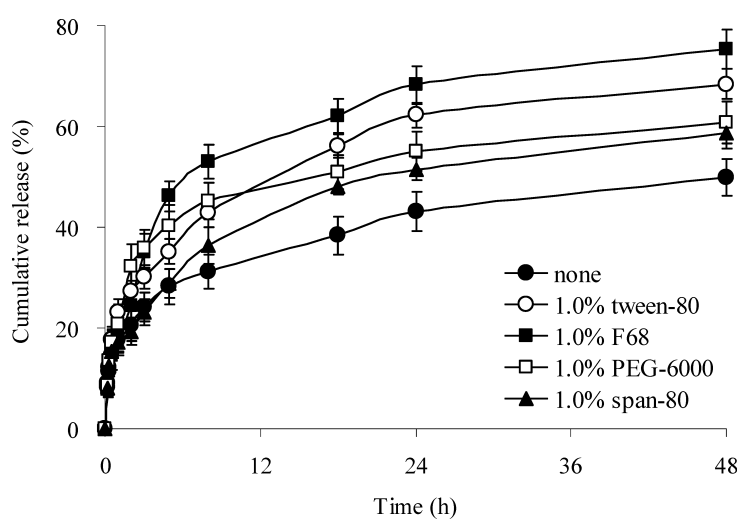

Fig. 5. Effect of Additives of Aqueous Phase on Release Profiles in Vitro

Types of additives were span-80, tween-80, F68 and PEG-6000. Other preparative conditions were controlled as follows: $\mathrm{pH} 4$ of $0.1 \% \mathrm{TPP}, 1 \mathrm{~h}$ of crosslinking time of $1.0 \% \operatorname{GTA}(n=3)$

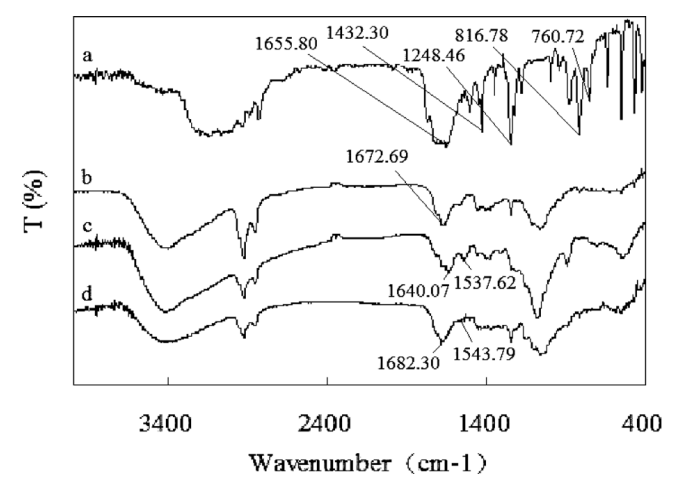

Fig. 6. FT-IR Spectra

(a) 5-FU, (b) 5-FU-loaded chitosan microspheres using GTA only, (c) 5-FU-loaded chitosan microspheres using TPP only, (d) 5-FU-loaded chitosan microspheres using both GTA and TPP. 


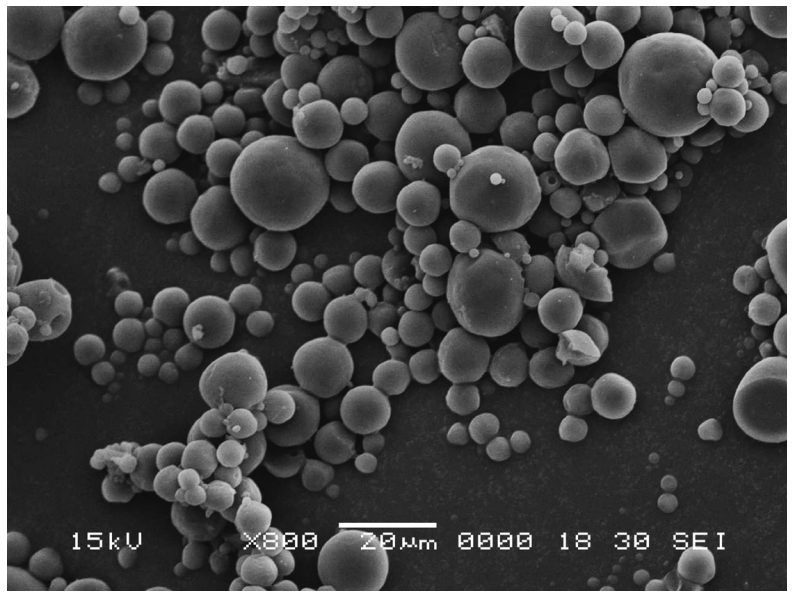

Fig. 7. SEM Picture of 5-FU-Loaded Chitosan Microspheres

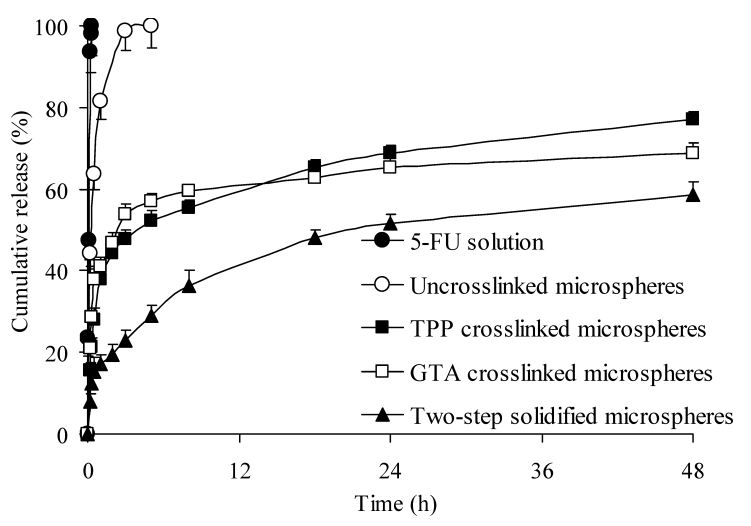

Fig. 8. Release Profiles of 5-FU-Loaded Chitosan Microspheres $(n=3)$

which was due to the reaction between $\mathrm{NH}_{2}$ groups of chitosan and $\mathrm{CHO}$ groups of GTA). Decreased adsorption of the characteristic absorption peaks of 5-FU was found $\left(1430.40,1247.18,813.81,760.22 \mathrm{~cm}^{-1}\right)$, indicating that 5FU had been efficiently entrapped in GTA crosslinked chitosan microspheres. For TPP crosslinked chitosan microspheres (Fig. 6c), due to the protonated amine groups formed on the chitosan molecule in ionic-crosslinking, absorption peaks of $1640.07 \mathrm{~cm}^{-1}$ and $1537.62 \mathrm{~cm}^{-1}$ were found. ${ }^{18,19)}$ When it came to the two-step solidified microspheres (Fig. 6d), the characteristic absorption peaks appeared at $1682.30 \mathrm{~cm}^{-1}(\mathrm{C}=\mathrm{N}$ stretching vibration $)$ and $1543.79 \mathrm{~cm}^{-1}$ (N-H stretching vibration of $\mathrm{NH}_{3}^{+}$group), due to GTA and TPP crosslinking reactions with chitosan in forming the drug-loaded microspheres.

SEM and release profile in vitro of the 5-FU-loaded chitosan microspheres prepared by two-step solidification under optimum condition ( $\mathrm{pH}$ value of TPP solution was 4, TPP concentration was $0.1 \%$, GTA concentration was $1.0 \%$, type of additive was span- 80 and the crosslinking time of GTA was $1 \mathrm{~h}$ ) are shown in Fig. 7 and Fig. 8. The microspheres were about $6.8 \mu \mathrm{m}$ in size and spherical in shape. Comparison of the release profiles of 5-FU solution of uncrosslinked microspheres, TPP crosslinked microspheres, GTA crosslinked microspheres and two-step solidified microspheres found that all the crosslinked microspheres had a sustained drug release effect. Application of both TPP and GTA during the preparation of microspheres resulted in the lowest burst rate (Fig. 8), showing that the two-step solidification method has great potential for the preparation of chitosan microspheres in a drug controlled-release system.

\section{Conclusion}

In this study, a novel two-step solidification method was applied to prepare the 5-FU-loaded chitosan microspheres. In the first solidification step, the chitosan emulsion was solidified by TPP to form microgel, and then was further crosslinked by GTA as a second solidification step. The preparation condition was optimized by using $0.1 \%$ TPP solution $(\mathrm{pH} 4.0)$ and $1.0 \%(\mathrm{v} / \mathrm{v})$ GTA with span- 80 additive under a crosslinking time of $1 \mathrm{~h}$ with TPP and GTA, respectively. The two-step solidification method fully overcame the disadvantages of poor morphology, low encapsulation efficiency and high burst effect in microsphere preparation when compared to the conventional crosslinking method. Encapsulation efficiency of 5-fluorouracil-loaded chitosan microspheres reached $78.5 \%$. The particle size distribution of all the chitosan microspheres was between $1-20 \mu \mathrm{m}$, and $58.7 \%$ of 5 -FU was released from the microspheres in a controlled release manner within a period of $48 \mathrm{~h}$. Hence, the two-step solidification method has a promising future for the controlled release of drugs. Further studies are underway to investigate this drug delivery system in an in vivo animal study.

Acknowledgements The authors acknowledge financial support from Shanghai Nanotechnology Leading Academic Discipline Foundation (No. 0852nm05900) and 111 Program of China (No. B07023).

\section{References}

1) Yang J. M., Su W. Y., Leu T. L., Yang M. C., J. Memb. Sci., 236, 3951 (2004).

2) Anal A. K., Stevens W. F., Remunan-Lopez C., Int. J. Pharm., 312 , 166-173 (2006).

3) Agnihotri S. A., Mallikarjuna N. N., Aminabhavi T. M., J. Controlled Release, 100, 5-28 (2004).

4) Akbuga J., Durmaz G., Int. J. Pharm., 111, 217-222 (1994)

5) Guo Q. H., Guo S. R., Wang Z. M., J. Controlled Release, 118, 318 324 (2007).

6) He W., Du Q., Cao D. Y., Xiang B., Fan L. F., Int. J. Pharm., 348, 3545 (2008)

7) Jameela S. R., Jayakrishnan A., Biomaterials, 16, 769-775 (1995).

8) Genta I., Perugini P., Conti B., Pavanetto F., Int. J. Pharm., 152, 237 246 (1997).

9) Genta I., Costatini M., Asti A., Conti B., Montanari L., Carbohydr. Polym., 36, 81-88 (1998).

10) Blanco M. D., Gomez C., Olmo R., Muniz E., Teijon J. M., Int. J. Pharm., 202, 29-39 (2000).

11) Wang L. Y., Gu Y. H., Su Z. G., Ma G. H., Int. J. Pharm., 311, 187195 (2006).

12) Wang L. Y., Gu Y. H., Zhou Q. Z., Ma G. H., Wan Y. H., Su Z. G., Colloids Surf. B: Biointerfaces, 50, 126-135 (2006).

13) Ko J. A., Park H. J., Hwang S. J., Park J. B., Lee J. S., Int. J. Pharm., 249, 165-174 (2002).

14) Gupta K. C., Jabrail F. H., J. Appl. Polym. Sci., 104, 1942-1956 (2007).

15) Shu X. Z., Zhu K. J., Eur. J. Pharm. Biopharm., 54, 235-243 (2002).

16) Aksungur P., Sungur A., Unal S., Iskit A. B., Squier C. A., Senel S., J. Controlled Release, 98, 269-279 (2004).

17) Aral C., Akbuga J., Int. J. Pharm., 168, 9-15 (1998).

18) Mi F. L., Sung H. W., Shyu S. S., J. Appl. Polym. Sci., 81, 1700-1711 (2001).

19) Mi F. L., Sung H. W., Shyu S. S., J. Polym. Sci. Part A: Polym. Chem., 38, 2804-2814 (2000). 